\title{
CHARACTERIZATION AND DISCRIMINATION OF THREE RHOPALOSIPHUM SPECIES (HOMOPTERA: APHIDIDAE) BASED ON ISOZYMES ${ }^{1}$
}

\author{
Sonia M. N. Lázzari ${ }^{2}$
}

\begin{abstract}
Seventeen clones of Rhopalosiphum padi (Linnaeus, 1758), 14 of R. maidis (Fitch, 1856), and two of $\mathbf{R}$. insertum (Walker, 1849), representing a wide range of host plants and geographic distribution, were examined electrophoretically to determine intra and interspecific variation. Twenty-one enzyme systems were tested using starch-gel techniques. The electromorph variation within species was low, as expected for parthenogenetic organisms. Frequency of heterozygotes was also relatively low for most populations. The percentage of polymorphic loci ranged from $0 \%$ to $27.3 \%$ in R. padi, but it was lower $(0 \%$ to $18.2 \%)$ in the completely anholocyclic R. maidis. No consistent correlation between band patterns and host plant or geographic origin could be established for R. padi and R. maidis. The distinction between $\mathbf{R}$. padi and $\mathbf{R}$. insertum was made by Est-1, Lap-2, Pgm, Got-1, and $\alpha$-gpdh. The enzymes Est-2, G-3pdh, Sdh, and Got-2 were useful to separate R. maidis from R. padi, while Est-1, Lap-2, Got-1, and $\alpha$-gpdh, in addition to the first three mentioned above, separated R. maidis from R. insertum. However, many other loci shared only one electromorph and had another that was useful to partially distinguish the species. The utilization of starch-gel electrophoresis as a taxonomic tool in distinguishing populations and species of aphids and its combination with other taxonomic methods are briefly discussed.
\end{abstract}

KEY WORDS. Rhopalosiphum, Aphididae, Homoptera, isozymes

Despite the worldwide economic importance of Rhopalosiphum species as pests of small grains, corn and other graminaceous crops, their taxonomic status and precise identification are still obscure (RICHARDS, 1960). Large phenotypic variability is observed within populations of Rhopalosiphum maidis (Fitchi, 1856), R. padi (Linnaeus, 1758), and R. insertum (Walker, 1849) (BLACKMAN \& EASTOP, 1984). On the other hand, the similarity between the apple-grain aphid, $R$. insertum and the bird cherry-oat aphid, $R$. padi, has caused many taxonomic confusion. Thus, traditional approaches based on morphological characters alone are unsatisfactory to clarify or solve such problems.

The use of electrophoretic techniques to assess either differences or similarities at the molecular level has become widespread for the study of aphid biotypes, species complexes, migration, and other ecological and taxonomic

1) Contribuição número 764 do Departamento de Zoologia, Universidade Federal do Paraná.

2) Departamento de Zoologia, Universidade Federal do Paraná, Caixa Postal 19020, 81531-970 Curitiba, Paraná, Brasil. 
problems. STEINER (1988) discussed the potential application of such techniques for aphid genetic studies.

TOMIUK \& WÖHRMANN (1983) analyzed electrophoretically 17 aphid species for 18 enzyme systems. They found a low degree of polymorphism, confirming investigations by WOOL et al. (1978), WÖHRMANN et al. (1978), and TOMIUK \& WÖHRMANN (1980). They constructed phylogenetic trees based on genetic identity and genetic distance and discussed the importance of comparing different methods to have reliable "molecular" trees. Rhopalosiphum padi and $R$. maidis, among other aphid species, were studied by SINGH \& CUNNINGHAM (1981) to determine genetic differences using starch-gel electrophoresis. They found esterases, leucine-alanine peptidase, acid phosphatase, and alkaline phosphatase patterns useful to distinguish species. The first two enzymes produced "diagnostic" patterns specific to individual species.

LOXDALE et al. (1983) found that the most useful enzymes for inter-generic and interspecific separation were: adenylate kinase (Adk), esterase (Est), glucose 6-phosphate dehydrogenase (G-6pdh), hexokinase (Hk), Malate dehydrogenase (Mdh), peptidase (Pep), phosphatase (Phos), phosphoglucomutase (Pgm), 6-phospho-gluconate dehydrogenase (6-Pgdh), and sorbitol dehydrogenase (Sdh). $R$. maidis and $R$. padi showed different patterns for Mdh-1 (apparently lacking in $R$. padi), peroxidase (Po), and especially Sdh. Pgm showed both intra and interspecific variation in staining intensity. In a later study LOXDALE et al. (1985b) observed that the use of serial one-dimensional polyacrylamide gel under different gel concentration and electrophoretic run time increased considerably the number of allozymes detected for peptidases.

Considerable differences in percentage of polymorphism from one year to the next and low average heterozygosity in populations of Sitobion avenae (Fabricius, 1775) were reported by LOXDALE et al. (1985a). The heterozygosity was confined mainly to the EST-1 locus. STEINER et al. (1985a) reported specific electromorph markers to assay variation in $R$. padi and $R$. maidis. No genetic differences between host-plant races were observed in $R$. padi. The low variation is interpreted as a function of parthenogenetic reproduction in aphids.

Isozymes were used by VOEGTLIN et al. (1987) to ascertain the source of annual immigrants of $R$. maidis into Illinois. Hk-1 was the only enzyme analyzed that showed geographic variation for the populations they studied.

Starch-gel electrophoresis was chosen for this study because it permits the analysis of a great number of samples and different enzyme systems in a short time, gives good resolution for population genetic studies, and is cheaper than other electrophoretic techniques.

The purposes of this study were to (1) investigate electromorph differences between species of Rhopalosiphum, focusing on isozymes useful to distinguish $R$. insertum from $R$. padi; (2) assess variation within species of 
Rhopalosiphum according to their host plant and geographic origin; and (3) generate information for studies comparing variation at the molecular and morphological levels and subsequent utilization of such data to construct identification keys and for other taxonomic studies.

\section{MATERIALS AND METHODS}

Seventeen clones of $R$. padi, 14 of $R$. maidis, two of $R$. insertum, and one of Schizaphis graminum (Rondani, 1852), used as an outgroup, were reared on barley (Hordeum vulgare Linnaeus, cv. Robust) in $12 \times 12 \times 3 \mathrm{~cm}$ clear plastic boxes covered with fine screen at $15^{\circ} \mathrm{C}$ and $18 \mathrm{~L}: 6 \mathrm{D}$ light regime. Each clone was derived from a single parthenogenetic female from samples collected on primary or secondary host plants, at different localities in the United States, as shown in table I. About one month before the tests, each population was recloned from a single female of each colony.

A total of 21 enzymes, coding for 22 gene loci were analyzed by starch-gel electrophoresis using two buffer systems as indicated in table II. The electrophoretic techniques, equipment, sample preparation, gel and buffer recipes, and staining procedures were based on STEINER \& JOSLYN (1979). The modifications from their work and particularities used in this study are indicated below.

About 30 apterous parthenogenetic females of each clone were placed in 1-dram screw-cap vials and stored at $-25^{\circ} \mathrm{C}$ prior to electrophoresis. Each electrophoretic sample consisted of two specimens ground with $25 \mu \mathrm{l}$ of dd water. The samples were applied to $4 \times 6 \mathrm{~mm}$ (Whatman no. 3) filter paper wicks and placed along the gel slot. The gels were made with $13 \%$ electrostarch poured in $6 \mathrm{~mm}$ molds $(350 \mathrm{ml})$. The gels were left to run overnight at $30 \mathrm{~mA}$ for both buffer systems. After electrophoresis, up to four slices were obtained from each gel and stained accordingly. For esterase the gel was presoaked in $50 \mathrm{ml} \mathrm{pH} 5.0$ boric solution before staining as indicated by STEINER \& JOSLYN (1979). The enzymes Ldh and Sdh were stained similar to Mdh, except that the D-L malate solution was replaced by D-L lactic acid to stain for Ldh and by sorbitol solution for Sdh staining ( $0.5 \mathrm{ml}$ sorbitol in $40 \mathrm{ml}$ dd water buffered with Trizma base to $\mathrm{pH}$ 7.0). The dipeptide used for Pep staining was L-leucyl-L-leucine, while the O-dianosidine dye was replaced by 2,6-dichlorophenol-indophenol which proved to be more efficient. The relative migration of each band in the gel was used as an indicator of genetic similarity and difference between populations. It was obtained in relation to the position of $R$. maidis bands, clone no. 10, since these were the most common electromorphs. These were arbitrarily assigned the value 1.00. Faster and slower electromorphs received values according to the difference in $\mathrm{mm}$ from this standard; faster being higher than 1.00 and slower smaller. An electromorph occurs at $100 \%$ frequency unless otherwise indicated. Each gel stained for a given enzyme was repeated at least three times and a minimal of 10 aphids of each population was analyzed for each system. 
Table I. Clones of Rhopalosiphum maidis, R. padi, R. insertum, and Schizaphis graminum, their host plant, site (USA) and date of collection. (After lab clonage, the aphids were reared on barley (Hordeum vulgare cv. Robust). See text for rearing procedure.

\begin{tabular}{|c|c|c|c|c|}
\hline SPECIES & $\begin{array}{c}\text { SAMPLE } \\
\text { No. } \\
\text { (CLONE) }\end{array}$ & HOST PLANT & SITE & $\begin{array}{l}\text { DATE } \\
\text { (M/D/YR) }\end{array}$ \\
\hline \multirow[t]{14}{*}{ R. maidis } & 1 & Corn [Zea mays L.] & Staples, MN & $08 / 27 / 87$ \\
\hline & 2 & Foxtail grass [Setaria sp.] & Urbana, IL & $07 / 10 / 87$ \\
\hline & 3 & Corn [Zea mays L.] & Parma, ID & $10 / 13 / 88$ \\
\hline & 4 & Corn [Zea mays L.] & Parma, ID & $09 / 26 / 87$ \\
\hline & 5 & Sweet corn [Zea mays L.] & Parma, ID & $09 / 26 / 87$ \\
\hline & 6 & $\begin{array}{l}\text { Barnyard grass [Echinochloa crusgalli (L.) } \\
\text { Beauvois] }\end{array}$ & Hidden Valley,ID & $08 / 20 / 87$ \\
\hline & 7 & $\begin{array}{l}\text { Barnyard grass [Echinochloa crusgalli (L.) } \\
\text { Beauvois] }\end{array}$ & Hidden Valley,ID & $08 / 27 / 87$ \\
\hline & 8 & Wheat [cv. Caldwell] [Triticum aestivum L.] & Parma, ID & $09 / 05 / 87$ \\
\hline & 9 & Corn [Zea mays L.] & Vicksburg, MS & $07 / 11 / 87$ \\
\hline & 10 & Corn [Zea mays L.] & Urbana, IL & $07 / 10 / 87$ \\
\hline & 23 & Corn [Zea mays L.] & Stillwater, OK & $05 / 30 / 87$ \\
\hline & 25 & Sorghum [Sorghum sp.] & Fayetteville, AR & $07 / 07 / 87$ \\
\hline & 29 & Corn [Zea mays L.] & Brookings, SD & $09 / 15 / 88$ \\
\hline & 30 & Sweet corn [Zea mays L.] & Brookings, SD & $09 / 15 / 88$ \\
\hline \multirow[t]{17}{*}{ R. padi } & 11 & Black cherry [Prunus serotina Ehrhart] & St. Paul, MN & $05 / 05 / 87$ \\
\hline & 13 & Barley [Hordeum vulgare L.] & Stillwater, OK & $01 / 08 / 88$ \\
\hline & 14 & Barley [Hordeum vulgare L.] & Salinas, CA & $01 / 06 / 88$ \\
\hline & 15 & Black cherry [Prunus serotina Ehrhart] & St. Paul, MN & $05 / 05 / 87$ \\
\hline & 16 & Barley [cv. Post] [Hordeum vulgare L.] & Perlsins, OK & $01 / 08 / 88$ \\
\hline & 17 & Wheat [cv. Largo] [Triticum aestivum L.] & Perlsins, OK & $01 / 08 / 88$ \\
\hline & 20 & Black cherry [Prunus serotina Ehrhart] & St. Paul, MN & $05 / 23 / 88$ \\
\hline & 21 & Spring wheat [Triticum aestivum L.] & St. Paul, MN & $05 / 28 / 88$ \\
\hline & 24 & Barley [Hordeum vulgare L.] & Purdue, IN & $06 / 29 / 88$ \\
\hline & 27 & Oats [Avena sativa L.] & Brookshire, TX & $06 / 16 / 88$ \\
\hline & 28 & Barley [Hordeum vulgare L.] & Brookings, SD & $09 / 15 / 88$ \\
\hline & 31 & Barley [Hordeum vulgare L.] & New Idaho, ID & $06 / 19 / 88$ \\
\hline & 32 & Wheat [Triticum aestivum L.] & Little River, AR & $03 / 02 / 89$ \\
\hline & 33 & Black cherry [Prunus serotina Ehrhart] & St. Paul, MN & $05 / 02 / 89$ \\
\hline & 34 & Barley [Hordeum vulgare L.] & St. Paul, MN & $05 / 21 / 89$ \\
\hline & 35 & Barley [Hordeum vulgare L.] & Urbana, IL & $04 / 06 / 89$ \\
\hline & 36 & Oats [Avena sativa, $\mathrm{L}]$. & Houston, TX & $05 / 01 / 89$ \\
\hline \multirow[t]{2}{*}{ R. insertum } & 22 & Barley [Hordeum vulgare L.] & Parma, ID & $07 / 01 / 88$ \\
\hline & 371 & Hawthorn [Crataegus L.] & Urbana, IL & $05 / 01 / 89$ \\
\hline S. graminum & $26 \quad I$ & Barley [Hordeum vulgare L.] & St. Paul, MN & $05 / 28 / 88$ \\
\hline
\end{tabular}


Table II. List of enzymes with respective enzyme classification code numbers (E.C.No.; International Union of Biochemistry Nomenclature), number of loci, and buffer systems used to analyze them in this study. ( ${ }^{*}$ System too weak or blurry to score).

\begin{tabular}{|c|c|c|c|}
\hline $\begin{array}{l}\text { BUFFER } \\
\text { SYSTEM }\end{array}$ & E. C. No. & ENZYME & $\begin{array}{l}\text { No. OF } \\
\text { LOCI }\end{array}$ \\
\hline \multirow[t]{9}{*}{$\mathrm{LiOH}$} & 3.1.3.2 & Acid phosphatase (Acph) & 1 \\
\hline & 1.2.3. 1 & Aldehyde oxidase (Aldox) & * \\
\hline & 3.1.3.1 & Alkaline phosphatase (Aph) & * \\
\hline & 3.1.1.1 & Esterase (Est) & 2 \\
\hline & 3.4.1.1 & Leucine aminopeptidase (Lap) & 2 \\
\hline & 1.1.1.40 & Malic enzyme (Me) & 1 \\
\hline & 3.4.1.1 & Peptidase (L-leucyl-L-leucine) (Pep) & 1 \\
\hline & 2.7.5.1 & Phosphoglucomutase (Pgm) & 1 \\
\hline & 5.3.1.9 & Phosphoglucose isomerase (Pgi) & 1 \\
\hline \multirow[t]{12}{*}{ CA-8 } & 2.7.4.3 & Adenylate kinase (Adk) & 2 \\
\hline & 4.1.2.13 & Aldolase (Ald) & * \\
\hline & 2.6.1.1 & Glutamate oxaloacetate transaminase (Got) & 2 \\
\hline & 1.2.1.12 & Glyceraldehyde-3-phosphate dehydrogenase (G-3pdh) & 1 \\
\hline & 1. 1.1 .8 & $\alpha$-glycerophosphate dehydrogenase ( $\alpha$-Gpdh) & 1 \\
\hline & 2.7.1.1 & Hexokinase (Hk) & 2 \\
\hline & 1. 1.1 .30 & Hydroxibutyrate dehydrogenase (Hbdh) & * \\
\hline & 1. 1.1 .42 & Isocitrate dehydrogenase (Idh) & 1 \\
\hline & 1. 1.1 .28 & Lactate dehydrogenase (Ldh) & * \\
\hline & 1.1.1.37 & Malic dehydrogenase (Mdh) & 2 \\
\hline & 1. 1.1 .44 & 6-phosphogluconate dehydrogenase (6-pgdh) & 1 \\
\hline & 1. 1.1 .14 & Sorbitol dehydrogenase (Sdh) & 1 \\
\hline
\end{tabular}

\section{RESULTS}

A few enzymes were found unsuitable for subsequent work under the conditions adopted in this study. Resolution problems were mainly due to low concentration of active enzymes in the samples. In this category were Aldox, Aph, Ald, Hbdh, and Ldh. Despite the good resolution, a few other loci had the same electromorph fixed in all populations and consequently they were not useful to distinguish between neither clones nor species. These included: Lap-1, Me, Pgi, Adk-1, Idh, and 6-pgdh; they were omitted from tables and figures, but considered in the analysis.

Intraspecific Variation. Table III shows a genetic comparison of $14 R$. maidis populations, listing the electromorph type of seven variable loci out of 22 examined. Most electromorph loci were fixed for a single most common type, designated as 1.00. The percentage of polymorphic loci ranged from $0 \%$ in most 
Table III. Electromorphs of seven variable loci in 12 populations of Rhopalosiphum maidis. A minimum of 10 aphids were analyzed from each population for each enzyme (Numbẹr in parenthesis represents percentage of frequency. Frequency is $100 \%$ when not indicated).

\begin{tabular}{|c|c|c|c|c|c|c|c|}
\hline $\begin{array}{c}\text { Population } \\
\text { No. }\end{array}$ & Pep & Pgm & Adk-2 & Got-1 & $\alpha$-gpdh & Hk-1 & Hk-2 \\
\hline 1 & 1.00 & 1.00 & $\begin{array}{l}1.00 \text { or } \\
1.00 / 1.02\end{array}$ & 1.00 & 1.00 & $\begin{array}{l}1.00(0.50) \\
1.00 / 1.04(0.50)\end{array}$ & $\begin{array}{l}1.00(0.25) \\
1.00 / 1.02(0.75)\end{array}$ \\
\hline 2 & 1.00 & 1.00 & $\begin{array}{l}1.00 \text { or } \\
1.00 / 1.02\end{array}$ & $\begin{array}{l}1.00(0.75) \\
1.00 / 0.98(0.25)\end{array}$ & 1.00 & $\begin{array}{l}1.00(0.50) \\
1.00 / 1.04(0.50)\end{array}$ & $\begin{array}{l}1.00(0.50) \\
1.00 / 1.02(0.50)\end{array}$ \\
\hline 3 & 1.00 & 1.00 & $\begin{array}{l}1.00 \text { or } \\
1.00 / 1.02\end{array}$ & 1.00 & 1.00 & $\begin{array}{l}1.00(0.50) \\
1.00 / 1.04(0.50)\end{array}$ & $\begin{array}{l}1.00(0.75) \\
1.00 / 1.02(0.25)\end{array}$ \\
\hline 4 & 1.00 & 1.00 & $\begin{array}{l}1.00 \text { or } \\
1.00 / 1.02\end{array}$ & 1.00 & 1.00 & $\begin{array}{l}1.00(0.50) \\
1.00 / 1.04(0.50)\end{array}$ & 1.00 \\
\hline 5 & 1.00 & 1.00 & $\begin{array}{l}1.00 \text { or } \\
1.00 / 1.02\end{array}$ & 1.00 & $\begin{array}{l}1.00(0.67) \\
1.00 / 0.98(0.33)\end{array}$ & $\begin{array}{l}1.00(0.50) \\
1.00 / 1.04(0.50)\end{array}$ & $\begin{array}{l}1.00(0.50) \\
1.00 / 1.02(0.50)\end{array}$ \\
\hline 6 & 1.00 & 1.00 & $\begin{array}{l}1.00 \text { or } \\
1.00 / 1.02\end{array}$ & 1.00 & 1.00 & $\begin{array}{l}1.00(0.50) \\
1.00 / 1.04(0.50)\end{array}$ & 1.00 \\
\hline 7 & 1.00 & 1.00 & $\begin{array}{l}1.00 \text { or } \\
1.00 / 1.02\end{array}$ & 1.00 & 1.00 & $\begin{array}{l}1.00(0.50) \\
1.00 / 1.04(0.50)\end{array}$ & 1.00 \\
\hline 8 & $1.00 / 0.98$ & 1.00 & $\begin{array}{l}1.00 \text { or } \\
1.00 / 1.02\end{array}$ & 1.00 & 1.00 & $\begin{array}{l}1.00(0.50) \\
1.00 / 1.04(0.50)\end{array}$ & $\begin{array}{l}1.00(0.50) \\
1.00 / 1.02(0.50)\end{array}$ \\
\hline 9 & 1.00 & 1.00 & $\begin{array}{l}1.00 \text { or } \\
1.00 / 1.02\end{array}$ & 1.00 & 1.00 & $\begin{array}{l}1.00(0.50) \\
1.00 / 1.04(0.50)\end{array}$ & 1.00 \\
\hline 10 & 1.00 & 1.00 & $\begin{array}{l}1.00 \text { or } \\
1.00 / 1.02\end{array}$ & 1.00 & 1.00 & $\begin{array}{l}1.00(0.50) \\
1.00 / 1.04(0.50)\end{array}$ & $\begin{array}{l}1.00(0.50) \\
1.00 / 1.02(0.50)\end{array}$ \\
\hline 23 & $1.00 / 0.98$ & $\begin{array}{l}1.00(0.67) \\
1.00 / 1.02(0.33)\end{array}$ & $\begin{array}{l}1.00 \text { or } \\
1.00 / 1.02\end{array}$ & 1.00 & 1.00 & $\begin{array}{l}1.00(0.50) \\
1.00 / 1.04(0.50)\end{array}$ & $\begin{array}{l}1.00(0.50) \\
1.00 / 1.02(0.50)\end{array}$ \\
\hline 25 & $1.00 / 0.98$ & $\begin{array}{l}1.00(0.67) \\
1.00 / 1.02(0.33)\end{array}$ & $\begin{array}{l}1.00 \text { or } \\
1.00 / 1.02\end{array}$ & 1.00 & 1.00 & $\begin{array}{l}1.00(0.50) \\
1.00 / 1.04(0.50)\end{array}$ & 1.00 \\
\hline 29 & $1.00 / 0.98$ & 1.00 & $\begin{array}{l}1.00 \text { or } \\
1.00 / 1.02\end{array}$ & 1.00 & 1.00 & $\begin{array}{l}1.00(0.50) \\
1.00 / 1.04(0.50)\end{array}$ & $\begin{array}{l}1.00(0.25) \\
1.00 / 1.02(0.75)\end{array}$ \\
\hline 30 & - & 1.00 & $\begin{array}{l}1.00 \text { or } \\
1.00 / 1.02\end{array}$ & 1.00 & $\begin{array}{l}1.00(0.67) \\
1.00 / 0.98(0.33)\end{array}$ & $\begin{array}{l}1.00(0.50) \\
1.00 / 1.04(0.50)\end{array}$ & 1.00 \\
\hline
\end{tabular}

clones to $18.2 \%$ in the Stillwater population from corn (no.23). A consistently heterozygous form at $\mathrm{Hk}-1$ was revealed for all populations in half of the gels stained for Hk. More than $50 \%$ of the clones also exhibited heterozygosity at Hk-2 in addition to the 1.00 electromorph. Other loci, such as Pep, Pgm, Got-1, and $\alpha$-gpdh, showed electromorph variation in at least one population. In all the cases the alternative forms were heterozygous with variable percentage of frequency within the clone. Adk-2 showed very broad bands, suggesting heterozygosity for all populations.

No correlation between the polymorphic loci and geographic origin or host plant could be established for $R$. maidis. Electromorphic variation, however, seemed to be greater in the more recent populations (clones 23, 25, 29,30 ) compared to those collected for more than one year before the tests (table I and table III).

Variation within $R$. padi is presented on table IV, showing variable electromorphs at nine out of the 22 examined loci. Mdh-1 and Mdh-2 were consistently polymorphic for all populations, with two alternative homozygous 
Table IV. Electromorphs of nine variable loci in 17 populations of Rhopalosiphum padi. A minimun of 10 aphids were analyzed from each population for each enzyme. (Number in parenthesis represents percentage of frequency. Frequency is $100 \%$ when not indicated). Not analyzed(-).

\begin{tabular}{|c|c|c|c|c|c|c|c|c|}
\hline $\begin{array}{l}\text { Population Acph-1 } \\
\text { No. }\end{array}$ & Pep & Adk-2 & Got-1 & $\alpha$-gpdh & Hk-1 & $\mathrm{Hk}-2$ & Mdh-1 & Mdh-2 \\
\hline $1.00 \quad 1$. & $1.00 / 0.98$ & $\begin{array}{l}1.00 \text { or } \\
1.00 / 1.02\end{array}$ & 1.00 & 1.00 & $\begin{array}{l}1.00(0.50) \\
1.00 / 1.04(0.50)\end{array}$ & 1.00 & $\begin{array}{l}1.00(0.50) \\
0.98(0.50)\end{array}$ & $\begin{array}{l}1.00(0.50) \\
0.98(0.50)\end{array}$ \\
\hline 1.001. & $1.00 / 0.98$ & $\begin{array}{l}1.00 \text { or } \\
1.00 / 1.02\end{array}$ & 1.00 & 1.00 & $\begin{array}{l}1.00(0.50) \\
1.00 / 1.04(0.50)\end{array}$ & $\begin{array}{l}1.00(0.20) \\
1.00 / 1.02(0.80)\end{array}$ & $\begin{array}{l}1.00(0.50) \\
0.98(0.50)\end{array}$ & $\begin{array}{l}1.00(0.50) \\
0.98(0.50)\end{array}$ \\
\hline $0.98 \quad 1$. & $1.00 / 0.98$ & 1.00 & $\begin{array}{l}1.00(0.75) \\
0.98(0.25)\end{array}$ & 1.00 & $\begin{array}{l}1.00(0.50) \\
1.00 / 1.04(0.50)\end{array}$ & $\begin{array}{l}1.00(0.40) \\
1.02(0.20) \\
1.00 / 1.02(0.40)\end{array}$ & $\begin{array}{l}1.00(0.50) \\
0.98(0.50)\end{array}$ & $\begin{array}{l}1.00(0.50) \\
0.98(0.50)\end{array}$ \\
\hline 1.00 & - & $\begin{array}{l}1.00 \text { or } \\
1.00 / 1.02\end{array}$ & 1.00 & 1.00 & $\begin{array}{l}1.00(0.50) \\
1.00 / 1.04(0.50)\end{array}$ & $\begin{array}{l}1.00(0.50) \\
1.00 / 1.02(0.50)\end{array}$ & $\begin{array}{l}1.00(0.50) \\
0.98(0.50)\end{array}$ & $\begin{array}{l}1.00(0.50) \\
0.98(0.50)\end{array}$ \\
\hline 1.00 & - & 1.00 & 1.00 & 1.00 & $\begin{array}{l}1.00(0.50) \\
1.00 / 1.04(0.50)\end{array}$ & 1.00 & $\begin{array}{l}1.00(0.50) \\
0.98(0.50)\end{array}$ & $\begin{array}{l}1.00(0.50) \\
0.98(0.50)\end{array}$ \\
\hline $1.00 \quad 1$. & 1.00 & $\begin{array}{l}1.00 \text { or } \\
1.00 / 1.02\end{array}$ & 1.00 & 1.00 & $\begin{array}{l}1.00(0.50) \\
1.00 / 1.04(0.50)\end{array}$ & 1.00 & $\begin{array}{l}1.00(0.50) \\
0.98(0.50)\end{array}$ & $\begin{array}{l}1.00(0.50) \\
0.98(0.50)\end{array}$ \\
\hline 1.00 & 1.00 & 1.00 & 1.00 & 1.00 & $\begin{array}{l}1.00(0.50) \\
1.00 / 1.04(0.50)\end{array}$ & 1.00 & $\begin{array}{l}1.00(0.50) \\
0.98(0.50)\end{array}$ & $\begin{array}{l}1.00(0.50) \\
0.98(0.50)\end{array}$ \\
\hline 1.00 & - & 1.00 & 1.00 & 1.00 & $\begin{array}{l}1.00(0.50) \\
1.00 / 1.04(0.50)\end{array}$ & $\begin{array}{l}1.00(0.50) \\
1.00 / 1.02(0.50)\end{array}$ & $\begin{array}{l}1.00(0.50) \\
0.98(0.50)\end{array}$ & $\begin{array}{l}1.00(0.50) \\
0.98(0.50)\end{array}$ \\
\hline 1.00 & 1.00 & 1.00 & 1.00 & $\begin{array}{l}1.00(0.50) \\
0.98(0.50)\end{array}$ & $\begin{array}{l}1.00(0.50) \\
1.00 / 1.04(0.50)\end{array}$ & 1.00 & $\begin{array}{l}1.00(0.50) \\
0.98(0.50)\end{array}$ & $\begin{array}{l}1.00(0.50) \\
0.98(0.50)\end{array}$ \\
\hline 1.00 & $1.00 / 0.98$ & 1.00 & 1.00 & 1.00 & $\begin{array}{l}1.00(0.50) \\
1.00 / 1.04(0.50)\end{array}$ & $\begin{array}{l}1.00(0.50) \\
1.02(0.50)\end{array}$ & $\begin{array}{l}1.00(0.50) \\
0.98(0.50)\end{array}$ & $\begin{array}{l}1.00(0.50) \\
0.98(0.50)\end{array}$ \\
\hline 1.001. & 1.00 & 1.00 & 1.00 & 1.00 & $\begin{array}{l}1.00(0.50) \\
1.00 / 1.04(0.50)\end{array}$ & 1.00 & $\begin{array}{l}1.00(0.50) \\
0.98(0.50)\end{array}$ & $\begin{array}{l}1.00(0.50) \\
0.98(0.50)\end{array}$ \\
\hline 0.98 & 1.00 & $\begin{array}{l}1.00 \text { or } \\
1.00 / 1.02\end{array}$ & 1.00 & $\begin{array}{l}1.00(0.50) \\
0.98(0.50)\end{array}$ & $\begin{array}{l}1.00(0.50) \\
1.00 / 1.04(0.50)\end{array}$ & $\begin{array}{l}1.00(0.50) \\
1.00 / 1.02(0.50)\end{array}$ & $\begin{array}{l}1.00(0.50) \\
0.98(0.50)\end{array}$ & $\begin{array}{l}1.00(0.50) \\
0.98(0.50)\end{array}$ \\
\hline 1.00 & $1.00 / 0.98$ & $\begin{array}{l}1.00 \text { or } \\
1.00 / 1.02\end{array}$ & 1.00 & 1.00 & $\begin{array}{l}1.00(0.50) \\
1.00 / 1.04(0.50)\end{array}$ & $\begin{array}{l}1.00(0.50) \\
1.00 / 1.02(0.50)\end{array}$ & $\begin{array}{l}1.00(0.50) \\
0.98(0.50)\end{array}$ & $\begin{array}{l}1.00(0.50) \\
0.98(0.50)\end{array}$ \\
\hline 1.00 & $1.00 / 0.98$ & 1.00 & 1.00 & 1.00 & $\begin{array}{l}1.00(0.50) \\
1.00 / 1.04(0.50)\end{array}$ & $\begin{array}{l}1.00(0.50) \\
1.00 / 1.02(0.50)\end{array}$ & $\begin{array}{l}1.00(0.50) \\
0.98(0.50)\end{array}$ & $\begin{array}{l}1.00(0.50) \\
0.98(0.50)\end{array}$ \\
\hline 1.00 & 1.00 & $\begin{array}{l}1.00 \text { or } \\
1.00 / 1.02\end{array}$ & 1.00 & 1.00 & $\begin{array}{l}1.00(0.50) \\
1.00 / 1.04(0.50)\end{array}$ & 1.00 & $\begin{array}{l}1.00(0.50) \\
0.98(0.50)\end{array}$ & $\begin{array}{l}1.00(0.50) \\
0.98(0.50)\end{array}$ \\
\hline 1.00 & $1.00 / 0.98$ & 1.00 & $\begin{array}{l}1.00(0.75) \\
1.00 / 0.98(0.25)\end{array}$ & 1.00 & $\begin{array}{l}1.00(0.50) \\
1.00 / 1.04(0.50)\end{array}$ & $\begin{array}{l}1.00(0.50) \\
1.00 / 1.02(0.50)\end{array}$ & $\begin{array}{l}1.00(0.50) \\
0.98(0.50)\end{array}$ & $\begin{array}{l}1.00(0.50) \\
0.98(0.50)\end{array}$ \\
\hline 1.00 & $1.00 / 0.98$ & 1.00 & $\begin{array}{l}1.00(0.75) \\
1.00 / 0.98(0.25)\end{array}$ & 1.00 & $\begin{array}{l}1.00(0.50) \\
1.00 / 1.04(0.50)\end{array}$ & $\begin{array}{l}1.00(0.50) \\
1.00 / 1.02(0.50)\end{array}$ & $\begin{array}{l}1.00(0.50) \\
0.98(0.50)\end{array}$ & $\begin{array}{l}1.00(0.50) \\
0.98(0.50)\end{array}$ \\
\hline
\end{tabular}

forms. Hk-1 was clearly heterozygous for all populations, but a very weak band was also observed throughout the populations. Polymorphism was also observed at Hk-2, Got-1, and $\alpha$-gpdh for some clones. Pep was heterozygous for at least half of the populations. A slow moving band at Acph-1 was detected for the westernmost populations (14 and 31). Similar to $R$. maidis, some clones of $R$. padi exhibited very broad bands at Adk-2, indicating heterozygotes, while a few others were narrow clearly indicating homozygotes.

The percentage of polymorphic loci ranged from $0 \%$ in most populations to $27.3 \%$ in the Salinas (no.14), Urbana (no.35), and Houston (no.36) clones. The Salinas population was highly polymorphic at $\mathrm{Hk}-2$, with two different 
homozygous bands and a third heterozygous form. It also showed uncommon electromorphs at Acph-1 and Got-1, shared only with the New Idaho population in the first case and with Urbana and Houston in the second. However, no consistent pattern between polymorphism and geographic origin or host plant could be established.

Only two populations of $R$. insertum were analyzed electrophoretically, but the results clearly showed that clone 22, as suspected, was a mixture of $R$. padi and $R$. insertum. The contamination was confirmed by chromosome examination and the clone was disregarded from this study. With only one clone left it was not possible to check electromorph variation among clones. However, variation within the same population was observed at $\mathrm{Hk}-2$. Half of the gels stained for this enzyme showed a sharp homozygous 1.00 band, the other half had an additional 1.02, slightly faster band. Heterozygotes were also detected at Hk-1, Got-2, and possibly Adk-2 (Fig. 1).

Interspecific Variation. The banding pattern on figure 1 represents the relative migration of electromorphs of the most variable loci over all the clones examined for each species. Several features of figure 1 concerning to intergeneric and interspecific variation are noteworthy. First, only seven enzymes showed different banding patterns between $S$. graminum and all the Rhopalosiphum species: Lap-2, Pep, Pgi, Adk-2, Sdh, Acph-1, and 6-pgdh. On the other hand, $S$. graminum shared an electromorph with all three Rhopalosiphum species at $40.9 \%$ of the 22 examined loci. Additionally it shared at least one electromorph with $R$. maidis at Pgm, Est-2, Got-2, and G-3pdh, making up $59.1 \%$ similarity between these two species. With $R$. insertum, electromorphs were shared at Est-1, Pgm, and Got-2 (54.5\%). They were shared with $R$. padi at Mdh-1 (45.5\%). Thus, the number of loci sharing electromorphs between the two genera increased to $68.2 \%$.

A second feature of figure 1 is the aspect of interspecific similarities and differences among Rhopalosiphum. None of the enzymes was suitable to distinguish between all the three species at once, although differences were found between any two species at several loci. The percentage of loci sharing electromorphs between $R$. insertum and $R$. padi was 77.3. The enzymes that showed different banding patterns for these two species were: Est-1, Lap-2, Pgm, Got-1, and $\alpha$-gpdh. The slow bands at Est-1 and Lap-2 and the fast ones at Got-1 and $\alpha$-gpdh seemed to be unique for insertum among Rhopalosiphum . At Pep, Got-2, Hk-1, Mdh-1, and Mdh-2 at least one allele was shared between $R$. insertum and $R$. padi, although the non-shared bands at these loci may confer some degree of genetic difference between the species.

The similarity decreased to $68.2 \%$ when comparing $R$. insertum with $R$. maidis (Fig.1), which is as low as the similarity between the two genera included in this study. The differentiating enzymes were: Est-1, Est-2, Lap-2, Got-1, $\alpha$-gpdh, G-3pdh, and Sdh. Partial electromorph differences were detected at Pgm, Pep, Got-2, and Hk-1. The banding pattern of $R$. maidis was more similar to $R$. padi than to $R$. insertum. The only enzymes that distinguished between $R$. 


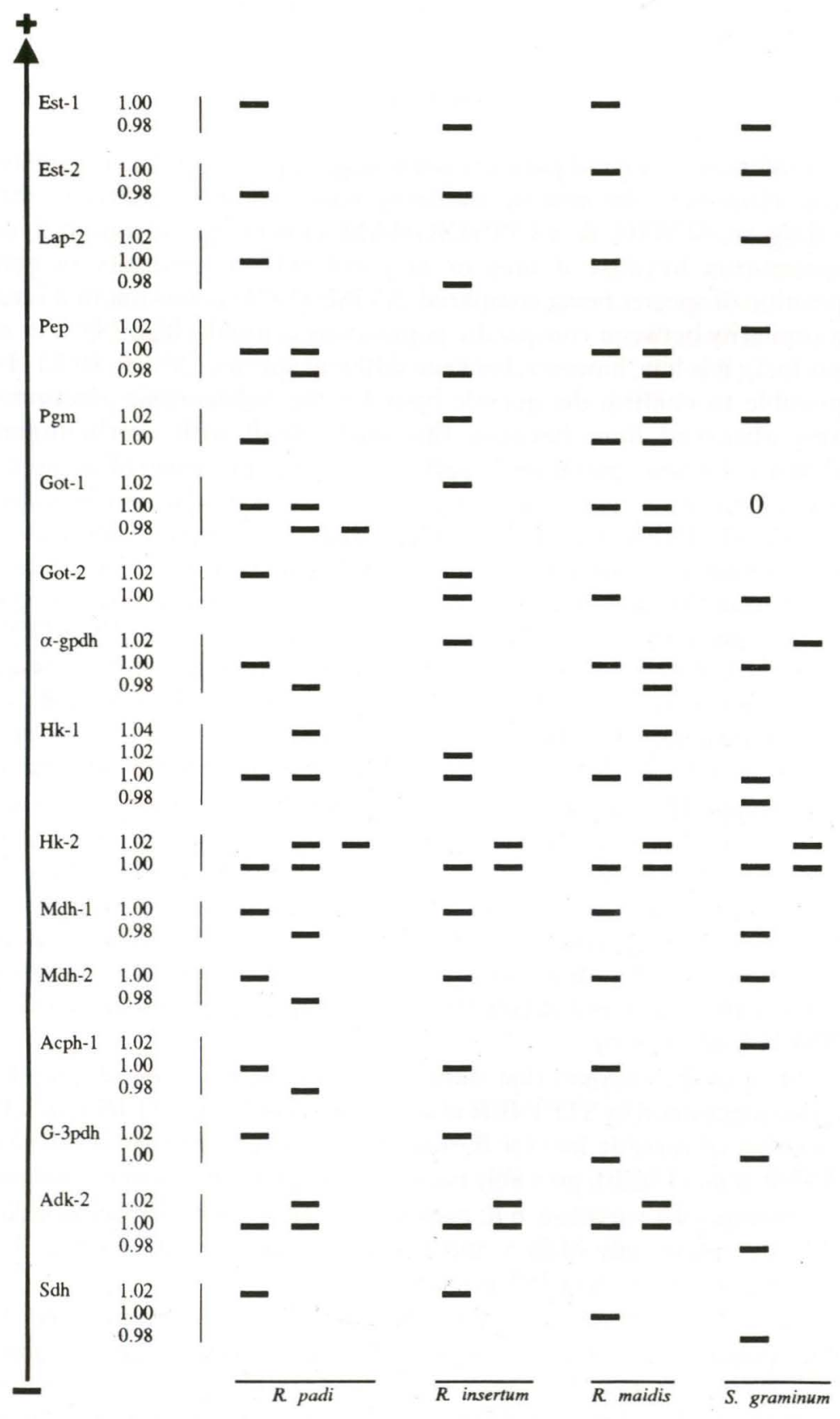

Fig. 1. Banding patterns of variable enzyme systems analyzed for three species of Rhopalosiphum and Schizaphis graminum. The relative migration for each band is given on the y-axis for each enzyme. Not analyzed (0). 
maidis and R. padi were: Est-2, Got-2, G-3pdh, and Sdh, although, Pgm, Mdh-1, Mdh-2, and Acph-1 also showed some bands not shared between them.

\section{DISCUSSION}

Differences in band patterns might suggest genetic differences between species. However, the genetic similarity based on band patterns should, according to SINGH \& CUNNINGHAM (1981), be interpreted as an overestimation because it may or may not reflect similarity in genetic composition of species being compared. AVISE (1974) points out that levels of genic similarity between conspecific populations is usually high ( $85 \%$ or more of their loci); it is low, however, between different species (20\% to $80 \%)$. It was not possible to confirm the genetic basis for the polymorphic electromorph systems observed here because this study dealt with parthenogenetic populations. Genetic variation is reflected by the presence of at least two electromorphs at a locus and it is expected to be low in parthenogenetic organisms (STEINER et al., 1985a). In this study, the complete anholocyclic $R$. maidis showed less polymorphic loci than R.padi that is subject to genetic recombination through sexual reproduction in most populations. The genetic variation found in table III for $R$. maidis is similar to that observed by STEINER et al. (1985b). Possible causes of genetic variation in aphid clonal lineages is discussed elsewhere (BLACKMAN, 1979). According to SELANDER (1976), many animal species populations are polymorphic at $25-50 \%$ of their enzyme loci, whereas individuals are, on average, heterozygous at 5-15\% of their loci.

Intraspecific Variation. Apparently fixed heterozygosity was observed at Hk-1 and probably at Hk-2 and Adk-2 throughout the species. Other loci, such as Pep, Pgm, Got-1, and $\alpha$-gpdh, also exhibited variable frequency of heterozygotes among the populations. STEINER et al. (1985a) suggested that heterozygosity has apparently been fixed via the parthenogenetic mode of reproduction. Those authors observed fixed heterozygosity for $\alpha$-gpdh in $R$. padi; it was also registered at Got-F for two $R$. maidis populations examined by STEINER et al. (1985b).

Most of the enzymes that showed variation within $R$. maidis in table III were also mentioned by STEINER et al. (1985b) and VOEGTLIN et al. (1987). The number of variable loci for $R$. maidis was higher than that in the work of STEINER et al. (1985b), probably because more populations were examined.

Intraspecific variation in $R$. padi was detected at several loci as indicated in table IV, while only Mdh-S and Pgm were polymorphic for the $R$. padi populations studied by STEINER et al. (1985a).

Lack of host plant and geographic origin dependent differences in the banding patterns was observed. STEINER et al. (1985a) suggested that this finding demonstrates that genetic differentiation between widespread populations may be a function of parthenogenesis and the clonal nature of aphid population founding. Hk-1 was found to be useful to indicate geographic variation in $R$. maidis by VOEGTLIN et al. (1987), but not here. 
Interspecific Variation. The genetic similarity between $S$. graminum and the Rhopalosiphum species was much higher in this study than that found by STEINER et al. (1985a); probably because the number of populations was much higher here and an additional species was examined. The number of loci sharing electromorphs increased within Rhopalosiphum compared to the intergeneric situation. This obviously reflects the genetic differences accumulated over evolutionary time.

Many enzymes in figure 1 were found to be useful to distinguish between Rhopalosiphum species agreeing with the list of taxonomically useful enzymes proposed by LOXDALE et al. (1983). However, Got and $\alpha$-gpdh that were very useful here to distinguish $R$. insertum from $R$. padi, were considered of little taxonomic use by those authors.

Although none of the enzymes could separate all three species at once, a few of them (Est-1, Lap-2, Got-1, and $\alpha$-gpdh) exhibited unique bands for $R$. insertum and several separated between any two species. The enzymes Hk-1, Pgi, Acph, and Ald were considered diagnostic and species specific for $R$. maidis and $R$. padi by STEINER et al. (1985a). However, they were of little use in this study; even Ald didn't work and Pgi and Acph separated only Rhopalosiphum from $S$. graminum.

At present there is no other study genetically comparing $R$. insertum and R. padi. The molecular data obtained here confirmed their taxonomic status as distinct species, despite the morphological similarity. They shared electromorphs at $77.3 \%$ of the examined loci, while the genetic similarity decreases to $68,2 \%$ when $R$. insertum is compared to the morphologically distinct $R$. maidis.

Comparing $R$. padi to $R$. maidis, the number of loci sharing electromorphs was relatively high $(81.8 \%)$. STEINER et al. (1985a) found $73.3 \%$ of similar loci between them. The higher value observed here probably reflects the larger number of clones examined. The banding patterns of Sdh, Got, Pep, Est, Pgm, Hk, and Adk as shown in figure 1 were very similar to those observed by LOXDALE et al. (1983) using PAGE. However, the results for Mdh disagreed with their observations and also from STEINER et al. (1985a). While $R$. padi lacked an additional band in their studies, $R$. maidis was the one that apparently lacked it here. On the other hand, SINGH \& CUNNINGHAM (1981) found two similar bands for them. The banding pattern for Pgm also differred from STEINER et al. (1985a), in which $R$. padi showed heterozygotes in some populations. Here heterozygosity was observed only for $R$. maidis.

The banding patterns of Est, Acph, Lap, and Me agreed with SINGH \& CUNNINGHAM (1981), who considered esterases the best enzymes for aphid species identification. In this study Est-1 distinguished $R$. insertum from $R$. padi and $R$. maidis, while Est-2 separated $R$. maidis from the others.

In conclusion, band patterns of several enzymes revealed by starch-gel electrophoresis were found to be useful to detect intra and interspecific genetic variation in Rhopalosiphum. Some loci seemed to be especially useful to 
distinguish $R$. insertum from $R$. padi, which have been taxonomically confused. Despite the satisfactory resolution of starch gel electrophoresis for population genetic studies, it seems that other additional electrophoretic techniques might help in the analysis of some enzyme systems. Overall results also indicated that more variation or similarity can be detected when larger numbers of populations from different host plants and geographic regions are examined. When one wants to find diagnostic loci, a large number of species or genera should be assessed. Although some enzymes may provide diagnostic loci for characterization and identification of species, electrophoretic data would be more valuable when supported by morphological and biological information in a multidisciplinary approach. Cluster analysis and resulting dendograms, that take into account gene frequency and genetic differences between populations, would also provide useful taxonomic information and shall be considered in further studies.

ACKNOWLEDGEMENTS. I am especially grateful to Dr. William W. Steiner, BCIRL-USDA, Columbia, Missouri, USA, for his advices on the electrophoretic technique and use of his lab facilities. This study was part of my $\mathrm{PhD}$ thesis at the University of Minnesota, MN, USA. It was partially supported by CAPES and UFPR (MEC - Brazilian government).

\section{BIBLIOGRAPHIC REFERENCES}

AVISE, J.C. 1974. Systematic value of electrophoretic data. Syst. Zool. 23: 465-481.

BLACKMAN, R.L. 1979. Stability and variation in aphid clonal lineages. Biol. J. Linn. Soc. 11: 259-277.

BLACKMAN, R.L. \& V.F. EASTOP. 1984. Aphids on the world's crops: an identificaton and information guide. J. Wiley \& Sons. Chichester, UK, 466p.

LOXDALE, H.D.; P. CASTAÑERA \& C.P. BROOKES. 1983. Electrophoretic study of enzymes from cereal aphid populations. 1. Electrophoretic techniques and staining systems for characterizing enzymes from six species of cereal aphids (Hemiptera: Aphididae). Bull. Entomol. Res. 73: 645-657.

LOXDALE, H.D.; I.J. TARR; C.P. WEBER; C.P. BROOKES; P.G.N. DIGBY \& P. CASTANERA. 1985a. Electrophoretic study of enzymes from cereal aphid populations. 3. Spatial and temporal genetic variation of populations of Sitobion avenae (F.) (Hemiptera: Aphididae). Bull. Entomol. Res. 75: 121-141.

LOXDALE, H.D.; J.A. RHODES \& J.S. FOX. 1985b. Electrophoretic study of enzymes from cereal aphid populations. 4. Detection of hidden genetic variation within populations of the grain aphid Sitobion avenae (F.) (Hemiptera: Aphididae). Theor. Appl. Genet. 70: 407-412.

RICHARDS, W.R. 1960. A synopsis of the genus Rhopalosiphum in Canada (Homoptera: Aphididae). Can. Entomol. Suppl. 13: 1-51. 
SELANDER, R.K. 1976. Genic variation in natural populations, p. 21-45. In: F. J. Ayala (ed.), Molecular Evolution. Sinauer Associates, Massachusetts.

SINGH, S.M. \& T.K. CUNNINGHAM. 1981. Morphological and genetic differentiation in aphids (Aphididae). Can. Ent. 113: 539-549.

STEINER, W.W.M. 1988. Electrophoretic techniques for the genetic study of aphids, p. 135-143. In: A.K. Minks \& P. Harrewijn (eds.), Aphids. Their biology, natural enemies and control. Vol. B. Elsevier Sc. Publ. Amsterdam.

STEINER, W.W.M. \& D.J. JOSLYN. 1979. Electrophoretic techniques for the genetic study of mosquitoes. Mosq. News 39: 35-54.

STEINER, W.W.M.; D.J. VOEGTLIN; M.E. IRWIN \& G. KAMPMEIER. 1985a. Electrophoretic comparison of aphid species: detecting differences based on taxonomic status and host plant. Comp. Biochem. Physiol. 81: 295-299.

STEINER, W.W.M.; D.J. VOEGTLIN; M.E. IRWIN. 1985b. Genetic differentiation and its bearing on migration in North American populations of the corn leaf aphid, Rhopalosiphum maidis (Fitch) (Homoptera: Aphididae). Ann. Entomol. Soc. Am. 78: 518-525.

TOMIUK, J. \& K. WÖHRMANN. 1980. Enzyme variability in populations of aphids. Theor. Appl. Genet. 57: 125-127.

. 1983. Enzyme polymorphism and taxonomy of aphid species. Z. zool. Syst. Evolutionsforsch. 21: 266-274.

VOEGTLIN, D.J., W.W.M. STEINER \& M.E. IRWIN. 1987. Searching for the source of the annual spring migrants of Rhopalosiphum maidis (Homoptera: Aphididae) in North America, p. 120-133. In: J. Holman, J. Pelikán, A.F.G. Dixon, \& L. Weismann (eds.), Population structure, genetics and taxonomy of aphids and Thysanoptera. Proc. Inter. Symp., Smolenice, Czechoslovakia. The Hague: SPB Academic Publishing.

WÖHRMANN, K., H.A. EGGERS-SCHUMACHER \& J. TOMIUK. 1978. Allozyme variation in natural populations of aphids. Proc. XIV Inter. Cong. Genetics, Moscow, Part I, 492p.

WOOL,D., S. BUNTING \& H.F. van EMDEN. 1978. Electrophoretic study of genetic variation in British Myzus persicae (Sulz.)(Hemiptera: Aphididae). Biochem. Genet. 16: 987-1006.

Recebido em 02XI.1992; aceito em 07.VI.1993. 